Journal Club

Editor's Note: These short, critical reviews of recent papers in the Journal, written exclusively by graduate students or postdoctoral fellows, are intended to summarize the important findings of the paper and provide additional insight and commentary. For more information on the format and purpose of the Journal Club, please see http://www.jneurosci.org/misc/ifa_features.shtml.

\title{
Proprioceptive Eye Position Signals Are Still Missing a Sensory Receptor
}

\author{
Hrishikesh M. Rao ${ }^{1}$ and Vincent Prevosto ${ }^{1,2}$ \\ ${ }^{1}$ Department of Biomedical Engineering and ${ }^{2}$ Center for Cognitive Neuroscience, Duke University, Durham, North Carolina 27708 \\ Review of Zimmermann et al.
}

To properly control body movements, the brain needs to keep track of body position. Visual and cognitive studies have paid particular attention to eye position signals, which are considered crucial to many aspects of visuomotor behavior and may help to maintain perceptual stability. A longstanding controversy surrounds the nature and origin of eye position signals, which could potentially derive, on the sensory side, from extraocular eye muscle (EOM) proprioceptors, and on the motor side, from a corollary discharge of velocity-to-position neuronal integrators, without precluding the possibility that both signals coexist (Donaldson, 2000). Eye position signals from brainstem neuronal integrators play an undisputed role in eye movement control, and pathways that carry those signals to cortical regions with eye positionmodulated activity have also been demonstrated (Prevosto et al., 2009). In contrast, whereas eye position signals have been found in primary somatosensory cortex (Wang et al., 2007), the paucity of knowledge on sensory organs and pathways for proprioceptive eye position signals has long put into question the relevance of EOM sensory inputs for visuomotor control.

It has been found that in most species, EOMs essentially lack classical proprio-

Received April 15, 2013; revised May 15, 2013; accepted May 20, 2013. We thank Marc Sommer for helpful comments on this manuscript. Correspondence should be addressed to Dr. Vincent Prevosto, Center for Cognitive Neuroscience, Duke University, Box 90999, Durham, NC 27708. E-mail: vincent.prevosto@duke.edu.

DOI:10.1523/JNEUROSCI.1594-13.2013

Copyright $\odot 2013$ the authors $\quad 0270-6474 / 13 / 3310585-03 \$ 15.00 / 0$ ceptors. In species where muscle spindles and Golgi tendon organs are found in EOMs, those sensory receptors are generally poorly developed (Maier et al., 1974; Donaldson, 2000). However, mammalian EOMs possess a unique structure, the palisade endings, which have long been posited to serve proprioceptive functions (Ruskell, 1978). EOMs differ from skeletal muscles in several respects. The nearly continuous activity of EOMs is carried out by specialized fatigue-resistant muscle fibers dominantly found in the muscle layer that connects to the orbit (orbital layer; Fig. 1). EOMs are also characterized by the presence of slow-contracting muscle fibers which do not propagate action potentials. Those fibers are innervated by multiple, "en grappe," terminals and consequently are called multiply innervated muscle fibers (MIFs). Palisade endings are found at the myotendinous (muscle-tendon) junction of MIFs that connect to the sclera, in the global layer of the EOMs (Fig. 1; Ruskell, 1978). Axons of palisade endings extend into the tendon, before turning around and splaying into a bundle of terminals oriented toward the muscle fibers. Their supposed role as specialized tendon organs has placed palisade endings as the main candidates for carrying proprioceptive information from EOMs. Clinical evidence further supports this hypothesis (Steinbach et al., 1987).

However, recent results have again brought into question the assumption that palisade endings are proprioceptive organs. Investigation of molecular pheno- types revealed that palisade endings are cholinergic structures (Blumer et al., 2009), and tracing studies located their cell body in close proximity to oculomotor nuclei (Lienbacher et al., 2011a; Zimmermann et al., 2011). In addition, immunoreactivity of nicotinic acetylcholine receptors by $\alpha$-bungarotoxin has shown that a substantial minority of palisade ending axons form sequential motor terminals along the muscle body before establishing tendinous terminations (Blumer et al., 2009).

In a recent follow-up experiment, Zimmermann et al. (2013) combined tracing and molecular analysis techniques to clarify motor and sensory features of palisade endings and axons giving rise to them. They confirmed that, like motoneurons, axons supplying palisade endings, as well as palisade endings themselves, are choline acetyltransferase immunoreactive. As a control, the authors showed that cholinergic neurons were absent in the trigeminal ganglion, the sensory ganglion typically assumed to relay proprioceptive signals from EOMs. Other motor features were also apparent. Immunohistochemistry targeted to calcitonin gene-related peptide (CGRP), a marker of neuronal activity systematically found in motoneurons supplying EOMs, consistently showed positive labeling in palisade endings and their axons. In addition, multilabeling experiments clearly showed that the cholinergic nerve fibers that supply palisade endings originate from neurons in the oculomotor motor 
nuclei and establish en grappe motor terminals along MIFs, in a manner similar to that of the usual motor innervation of MIFs (Fig. 1). This finding runs contrary to the suggestion made that neurons giving rise to palisade endings and multiple motor contacts, respectively, form two distinct populations (Lienbacher et al., 2011b).

Together, results from the present study indicate that motor features dominate in palisade endings and particularly in the axons supplying them. Nonetheless, an ambiguity remains about the relative proportion of nerve fibers that form palisade endings which also form motor terminals on MIFs. Examination of axonal progress along the muscle fibers is limited by topological constraints, so assessments of the number of palisade ending axons that have motor terminals on MIFs are likely underestimates. Unfortunately, the authors did not provide an additional analysis based on examination of tracerpositive populations. Therefore, it remains unclear whether most axons would be expected to make motor contacts on MIFs, as the summary schema suggests (Zimmermann et al., their Fig. 8 ), or whether palisade ending innervation should be subdivided into two separate groups.

Despite thorough immunohistochemical examination, palisade endings remain surprisingly difficult to classify. Molecular features, such as positive CGRP reactivity, support the notion that palisade endings are a motor structure. However, they retain structural features similar to Golgi tendon organs. Accordingly, the lack of $\alpha$-bungarotoxin immunoreactivity in synapses formed by palisade endings at the tendinous terminals is inconsistent with a motor role. In addition, the small number of palisade ending terminals that do contact a muscle fiber lack a basal membrane, like other sensory nerve terminals. A complementary study using a retrograde instead of an anterograde tracer, aimed at defining molecular characteristic of palisade ending cell bodies, would help solve conflicting findings that palisade ending axons have motor features, while palisade ending cell bodies appear to have morphological features closer to sensory neurons (Lienbacher et al., 2011b).

The role of palisade endings remains largely open to speculation. Although the proximal portions of some axons that terminate in palisade endings contact muscle fibers, most palisade endings do not make such contacts themselves, nor do they resemble classical motor terminations. The

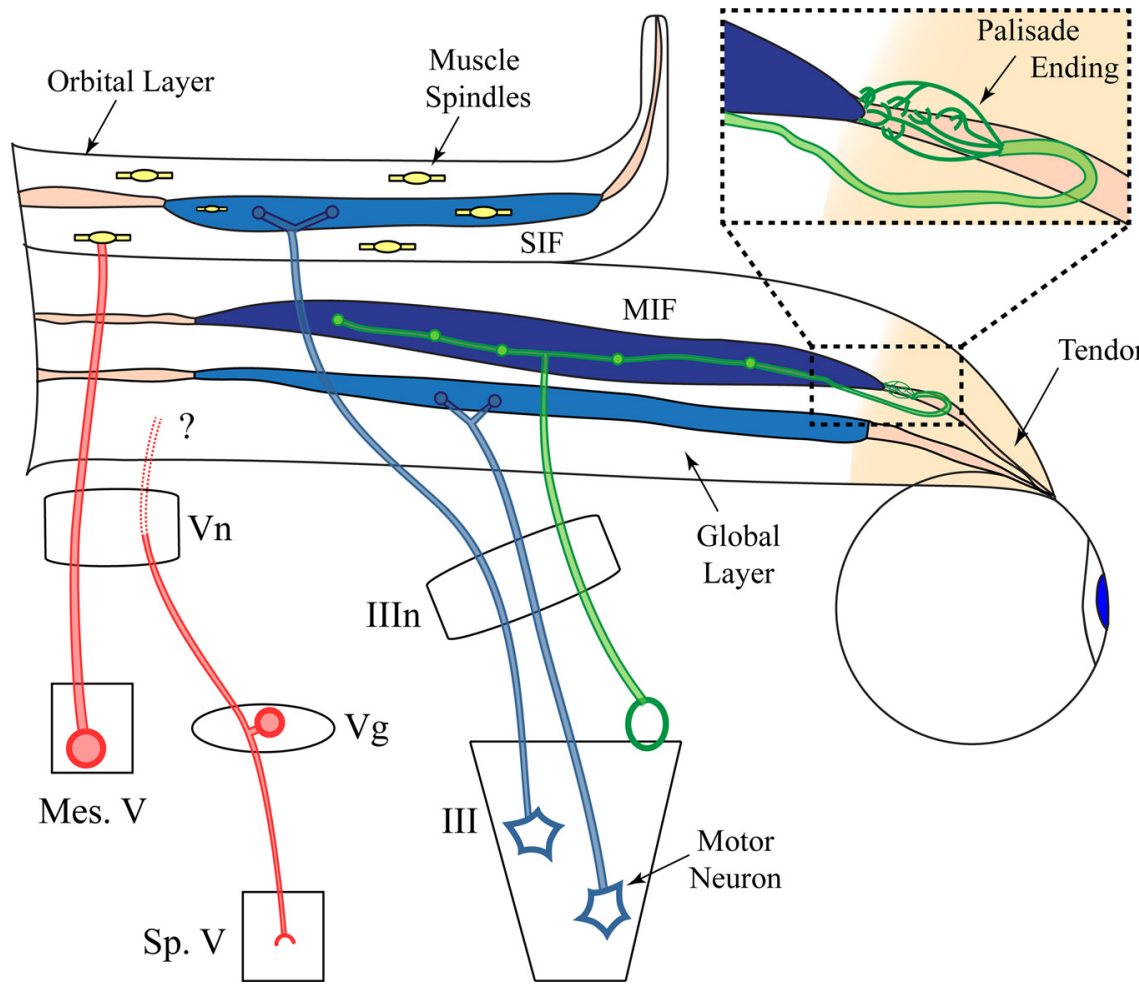

Figure 1. Schematic morphology of extraocular muscles with motor (blue) and sensory (red) innervation. Axons supplying palisade endings (green) stem from the periphery of the motor nucleus innervating a given EOM (here oculomotor nucleus to medial rectus). Some palisade ending nerve fibers form contacts on the MIFs (dark blue). Singly innervated fibers (SIF, "fast" fibers), and muscle spindles (when present), are predominantly found in the orbital layer. Inset, Palisade ending termination at the myotendinous junction. III, Oculomotor nucleus; IIIn, third cranial nerve; Vn, trigeminal nerve; Vg, trigeminal ganglion; Sp. V, spinal trigeminal nucleus; Mes. V, mesencephalic trigeminal nucleus.

potential action of acetylcholine on collagen fibrils at these junctions is also unknown. Except to postulate the occurrence of unidentified $\alpha$-bungarotoxin-negative acetylcholine receptors in these synapses, the likelihood that palisade endings transmit contractile inputs to the myotendinous junction seems remote.

Considering that palisade endings are ubiquitous among mammalian EOMs, unlike other proprioceptors, their function is surely evolutionarily advantageous. Therefore, however minute the actions that palisade endings may exert, we would expect them to be behaviorally relevant. Taking into account their location in the orbital layer, their association with the myotendinous junction, and the location of their cell body in proximity with structures associated to vergence and accommodation, a parsimonious hypothesis has gained ground: that palisade endings contribute to the fine control of ocular alignment, a suggestion that conveniently satisfies both proponents of a sensory and of a motor role (Lienbacher et al., 2011b; Zimmermann et al. 2013).

If palisade endings cannot provide the requisite signals needed to produce the proprioceptive representation of eye position found in primary somatosensory cortex, what are the relevant pathways? The composite nature of cortical eye position signals provides a clue: signals from multiple EOMs appear to be combined through a multisynaptic ascending pathway, to generate a delayed, omnidirectional representation of eye position (Wang et al., 2007). The notion that specialized and poorly developed receptors, which compose the more traditional sensory input to trigeminal pathways (Porter, 1986; Wang and May, 2008), may also contribute to the proprioceptive sense of eye position, perhaps in complement to palisade endings, is compatible with this organization. However, their contribution to sensorimotor transformations in cortical circuits is unlikely, as it has been shown that trigeminal nucleus and cuneate nucleus recipient regions of EOM sensory inputs do not project to areas of the posterior parietal cortex with demonstrated eye position gain fields (Prevosto et al., 2011).

A number of additional experiments are needed to tackle remaining questions about the role of palisade endings. In ad- 
dition to further molecular profiling of the somata of palisade endings as suggested above, tracing experiments can be designed to obtain a complete characterization of central projections. Using a conventional tracer such as biotinylated dextran amine, injections in the myotendinous junction would not only label a palisade ending cell body, but also the potential collateral targets among brainstem eye movement control circuits. Now that neurons innervating palisade endings have been firmly located in the periphery of motor nuclei, electrophysiological recordings may succeed at elucidating their function. Finally, experiments using optogenetic tools may achieve pathwayspecific manipulation of activity, which would yield a better understanding of the influence of palisade endings on peripheral as well as central systems, including cortical regions.

\section{References}

Blumer R, Konakci KZ, Pomikal C, Wieczorek G, Lukas JR, Streicher J (2009) Palisade endings: cholinergic sensory organs or ef- fector organs? Invest Ophthalmol Vis Sci 50: 1176-1186. Medline

Donaldson IM (2000) The functions of the proprioceptors of the eye muscles. Philos Trans $\mathrm{R}$ Soc Lond B Biol Sci 355:1685-1754. CrossRef Medline

Lienbacher K, Mustari M, Ying HS, BüttnerEnnever JA, Horn AK (2011a) Do palisade endings in extraocular muscles arise from neurons in the motor nuclei? Invest Ophthalmol Vis Sci 52:2510-2519. CrossRef Medline

Lienbacher K, Mustari M, Hess B, BüttnerEnnever J, Horn A (2011b) Is there any sense in the Palisade endings of eye muscles? Ann N Y Acad Sci 1233:1-7. CrossRef

Maier A, DeSantis M, Eldred E (1974) The occurrence of muscle spindles in extraocular muscles of various vertebrates. J Morphol 143: 397-408. CrossRef Medline

Porter JD (1986) Brainstem terminations of extraocular muscle primary afferent neurons in the monkey. J Comp Neurol 247:133-143. CrossRef Medline

Prevosto V, Graf W, Ugolini G (2009) Posterior parietal cortex areas MIP and LIPv receive eye position and velocity inputs via ascending preposito-thalamo-cortical pathways. Eur J Neurosci 30:1151-1161. CrossRef Medline

Prevosto V, Graf W, Ugolini G (2011) Proprioceptive pathways to posterior parietal areas MIP and LIPv from the dorsal column nuclei and the postcentral somatosensory cortex.
Eur J Neurosci 33:444-460. CrossRef Medline

Ruskell GL (1978) The fine structure of innervated myotendinous cylinders in extraocular muscles of rhesus monkeys. J Neurocytol 7:693-708. CrossRef Medline

Steinbach MJ, Kirshner EL, Arstikaitis MJ (1987) Recession vs marginal myotomy surgery for strabismus: effects on spatial localization. Invest Ophthalmol Vis Sci 28:1870-1872. Medline

Wang N, May P (2008) Peripheral muscle targets and central projections of the mesencephalic trigeminal nucleus in macaque monkeys. Anat Rec (Hoboken) 291:974-987. CrossRef

Wang X, Zhang M, Cohen IS, Goldberg ME (2007) The proprioceptive representation of eye position in monkey primary somatosensory cortex. Nat Neurosci 10:640-646. CrossRef Medline

Zimmermann L, May PJ, Pastor AM, Streicher J, Blumer R (2011) Evidence that the extraocular motor nuclei innervate monkey palisade endings. Neurosci Lett 489:89-93. CrossRef Medline

Zimmermann L, Morado-Díaz CJ, Davis-López de Carrizosa MA, de la Cruz RR, May PJ, Streicher J, Pastor ÁM, Blumer R (2013) Axons giving rise to the palisade endings of feline extraocular muscles display motor features. J Neurosci 33:2784-2793. CrossRef Medline 